\title{
Uso de un velocímetro de efecto acústico para la medición del campo de velocidad en un flujo de agua
}

\author{
Delgado, Miguel \\ Universidad Tecnológica de Panamá \\ Panamá, Panamá \\ miguel.delgado3@utp.ac.pa \\ Smith, Michael \\ Universidad Tecnológica de Panamá \\ Panamá, Panamá \\ michael.smith@utp.ac.pa \\ Pulido, Gonzalo \\ Universidad del Istmo \\ Panamá, Panamá \\ gpulido@udelistmo.edu
}

\begin{abstract}
Measurement of field velocities in water flows is a recurring need in water resources studies, analysis of hydraulic phenomena, and turbulent flow problems. In three-dimensional flow studies, it is required to measure the three components of velocity at the point of measurement, not to alter the flow at that point, and to record temporal variations in velocity. The Acoustic Doppler velocimeter (ADV) meets these three conditions. This research reports the use and implementation in the field of an ADV manufactured by NORTEK, model Vectrino Profiler. After understanding the use of the equipment and its software, an installation was developed to measure the velocities in the nodes of a $20 \times 20$ $\mathrm{cm}$ mesh, built in a vertical section of a pre-flocculation unit in a water purification plant. The sophistication of the instrument allows a large amount of data to be recorded for each measurement point, but this has the drawback of requiring a lot of post-processing to finally obtain the velocity components at the measurement point. The research question is whether the velocity components displayed on the screen during a steady-state measurement are statistically similar to those resulting from post-processing. An affirmative answer was found, which greatly simplifies the use of this equipment for full-scale field measurements in stable flow applications.
\end{abstract}


Keywords: Vectrino Profiler, acoustic measurements, velocity field, SURFER, Acoustic Doppler Velocimeter.

\section{Resumen}

La medición del campo de velocidades en flujos de agua es una necesidad recurrente en estudios de recursos hídricos, análisis de fenómenos hidráulicos y problemas de flujo turbulento. En estudios de flujos tridimensionales se requiere medir las tres componentes de velocidad en el punto de medición, no alterar el flujo en dicho punto y registrar las variaciones temporales de la velocidad. El Velocímetro Acústico Doppler (ADV, por sus siglas en inglés), cumple estas tres condiciones. En esta investigación se reporta el uso e implementación en campo de un ADV fabricado por NORTEK ${ }^{\circledR}$, modelo Vectrino Profiler. Después del entendimiento del uso del equipo y su software, se desarrolló una instalación para medir las velocidades en los nodos de una malla de $20 \times 20 \mathrm{~cm}$, construida en una sección vertical de una unidad de prefloculación en una planta potabilizadora de agua. La sofisticación del instrumento permite registrar una gran cantidad de datos para cada punto de medición, pero esto tiene el inconveniente de requerir mucho posprocesamiento para obtener finalmente las componentes de velocidad en el punto de medición. La pregunta de investigación es si las componentes de la velocidad mostradas en pantalla durante una medición en estado estable son estadísticamente similares a las resultantes del posprocesamiento. Se encontró respuesta afirmativa a escala real, lo que simplifica notoriamente el uso de este equipo para mediciones de campo a escala real, en aplicaciones de flujo estable.

Palabras claves: Vectrino Profiler, medidas acústicas, campo de velocidad, SURFER, Velocímetro Doppler Acústico.

\section{INTRODUCCIÓN}

Uno de Los parámetros más importantes en el diseño de las obras hidráulicas es el campo de velocidades, es decir, poder representar con la mejor exactitud posible el vector velocidad, teniendo en cuenta sus variaciones en el espacio y en el tiempo. Con los años se han desarrollado diversos métodos e instrumentos para tal fin. Desde el siglo XX se han usado correntómetros mecánicos, con algunas importantes limitaciones: A) solo mide una componente de velocidad, B) no registran las variaciones temporales de velocidad (a menos que cuenten con un registrador de datos) y C) al colocar el instrumento en el punto 
donde se desea medir la velocidad, altera el flujo [1]. En décadas recientes ha incrementado notablemente el desarrollo y uso de Velocímetros de Efecto Acústico (ADV), que resuelven las tres limitantes anteriores [2], [3].

Como parte del proyecto se requiere medir el campo de velocidades en una sección de prueba en la planta potabilizadora Jaime Díaz Quintero de la Chorrera con el objetivo de comparar los datos obtenidos con el software Vectrino Profiler vs los posprocesados en MATLAB y desarrollar mapas de contorno, mediante el uso del programa SURFER con los registros de las velocidades obtenidas.

\section{EQUIPO}

En esta investigación se utilizó un Vectrino Profiler (ADV) producido por la empresa NORTEK, el equipo cuenta con una sonda de acero inoxidable que tiene 4 transductores de recepción y un transductor de transmisión de muy alta resolución, utilizado para realizar mediciones de velocidad en un volumen de muestreo de $30 \mathrm{~mm}$ que genera datos tridimensionales de velocidad con una resolución de hasta $1 \mathrm{~mm}$ y una tasa de muestreo de hasta $100 \mathrm{~Hz}$ [4].

Consideraciones para su correcto funcionamiento entregadas por NORTEK [5]:

1. Revisar que no haya una obstrucción entre el sensor y el punto focal (volumen de medición) ubicado a unos $5 \mathrm{~cm}$ por debajo de los transductores.

2. Los transductores acústicos deben estar sumergidos, durante la toma de datos. Operar el Vectrino Profiler cuando los transductores están fuera del agua no causará ningún daño, pero los datos nos tendrán sentido.

3. Asegurarse que la estructura de montaje es estable ya que pequeñas vibraciones de está puede generar grandes aceleraciones y las velocidades obtenidas se verán afectadas.

4. La calidad de los datos recolectados dependerá de que haya suficientes partículas en suspensión.

5. La mejor calidad de obtención de datos se logra cuando el flujo principal es perpendicular al eje de transmisión, se debe evitar el flujo directo al eje de transmisión.

Se tomaron en consideración también los estudios de eliminación de picos [6] y la de reducción de ruido [7] para mejorar las estadísticas de velocidades medidas a diferentes frecuencias de muestreo. 


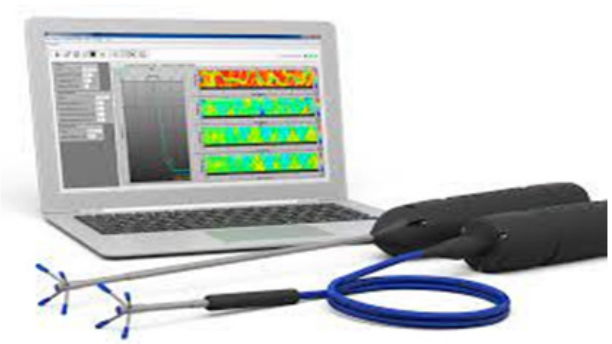

Figura 1. Software del Vectrino Profiler.

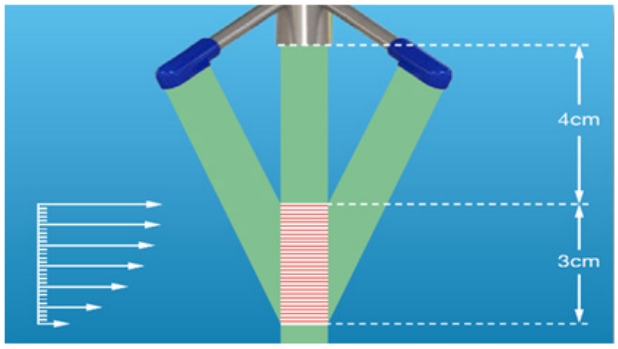

Figura 2. Proyección del velocímetro a través de los transductores.

\section{METOdOLOGÍA}

Para efectuar el estudio se eligió una sección vertical bidimensional dispuesta perpendicularmente en la unidad de prefloculación de la potabilizadora; la sección elegida es de $210 \mathrm{~cm}$ de ancho y $360 \mathrm{~cm}$ de profundidad. Se idealizo una cuadricula de $20 \times 20 \mathrm{~cm}$ para medir el campo de velocidades y el ADV se configuró para tomar lecturas con una frecuencia de $10 \mathrm{~Hz}$, en un perfilado de 8 celdas con un tamaño de $4 \mathrm{~mm}$ cada una [5]. Los datos obtenidos de cada medición son almacenados por el computador conectado al ADV y exportados vía MATLAB para su posterior reducción, el cual es un proceso largo, que solo se justifica para realizar estudios sofisticados de la turbulencia. Para cada punto se evaluó usar los datos tridimensionales de la velocidad que aparece en la ventana del software del Vectrino y comparar con las mediciones de las tres componentes de velocidad en el punto de interés procesando los datos registrados por el ADV a través del software MATLAB para el procesamiento completo de los datos. Los contornos de velocidad en la sección de análisis se obtuvieron mediante el software SURFER para ambos casos analizados.
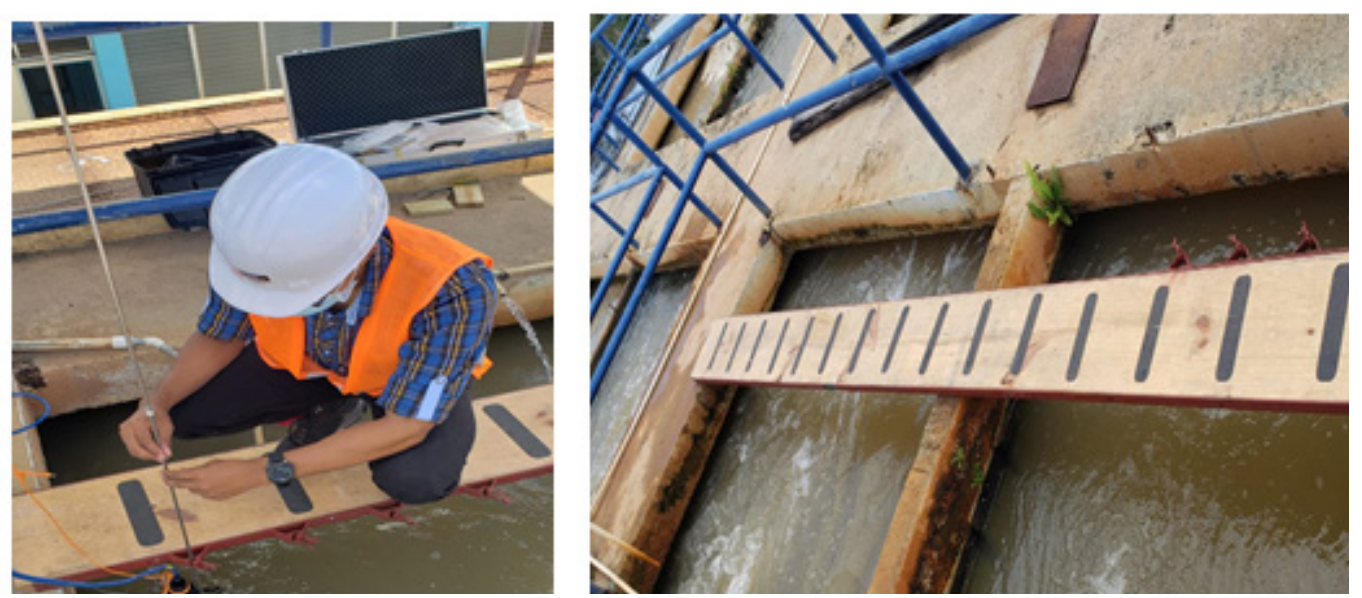

Figura 3. Instalación experimental para uso del ADV en el proyecto de la potabilizadora. 


\section{RESULTADOS}

Se compararon los datos de velocidad capturadas en pantalla vs las posprocesadas en MATLAB mediante una hoja de Excel, observándose que de los 459 datos de velocidad solo 25 (5.45\%) de ellas dieron diferencias por encima del $5 \%$. Respecto al porcentaje de los datos que dieron un error mayor a $5 \%$ un $68 \%$ de las 25 muestras se dieron porque se sobrepasó el límite de resolución del Vectrino Profiler, que tiene una exactitud de $\pm 1 \mathrm{~mm} / \mathrm{s}$ y aquellas velocidades estaban por debajo de la capacidad de lectura del instrumento. Luego se realizaron mapas de contorno de ambos métodos se puede observar que las gráficas son semejantes y las variaciones no son apreciables. Ver figura 4 y 5.

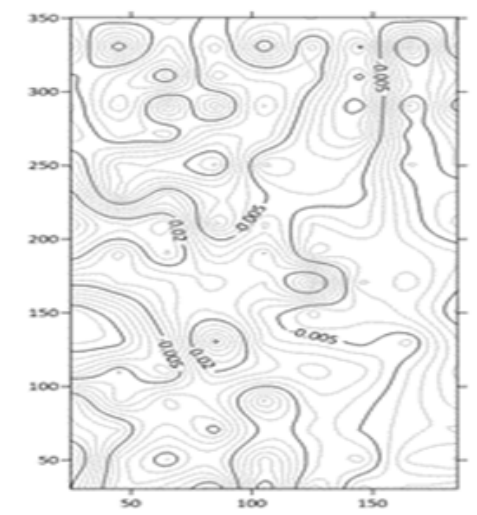

Figura 4. Mapa de contorno de datos extraídos de pantalla.

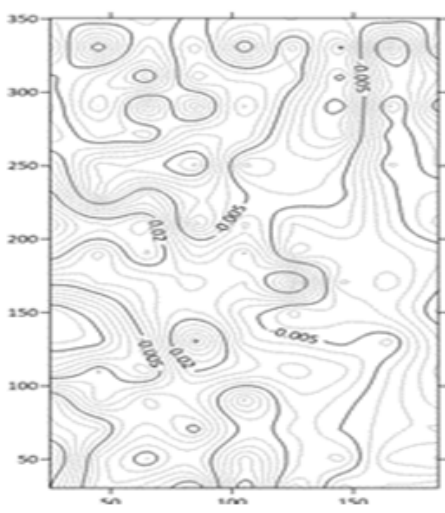

Figura 5. Mapa de contorno de datos procesados en MATLAB.

\section{CONCLUSIONES}

El Vectrino Profiler es un ADV que permite medir los campos de velocidades, no solo en el proyecto referido aquí, consistente en mediciones estables en secciones transversales de unidades de una planta potabilizadora sino para estudios que requieren capturar de manera mucho más detallada los campos de flujo y a su vez poder observar de forma gráfica el comportamiento de las tres componentes de velocidad dentro de la unidad en estudio.

Con base a los valores obtenidos en esta evaluación, la toma directa de datos en pantalla con el software Vectrino Profiler es estadísticamente valida y agiliza el proceso de la toma de velocidades lo cual permite que este sea un equipo confiable para el análisis del comportamiento de un flujo.

\section{Agradecimiento}

A la Secretaría Nacional de Ciencia, Tecnología e Innovación (SENACYT) por el financiamiento del proyecto "Formulación de la optimización de una planta potabilizadora 
mediante modelación matemática y calibración en situ", adjudicado a la Universidad del Istmo bajo el contrato IOMA 19-006, del cual se desprende esta investigación. Además, al Instituto de Acueductos y Alcantarillados Nacionales (IDAAN) por el permanente apoyo brindado en el desarrollo de la investigación.

\section{Referencias}

[1] G. Tapia Rodríguez, J. P. Molina Aguilar, G. B. Pérez Morales, and A. A. Torres Acosta, "Metodología para la medición de la velocidad de flujo en un río en el diagnóstico de la socavación en pilas de un puente, utilizando un dispositivo electrónico," Instituto Mexicano del Transporte, 2012.

[2] F. Homayounfar and B. Khorsandi, "Application of post-processing methods on the velocity statistics measured at different sampling frequencies using acoustic Doppler velocimeter," J. Hydraul., vol. 0, Jan. 2021, doi: 10.30482/JHYD.2021.257224.1487.

[3] Nortek, "The Comprehensive Manual for Velocimeters," Nortek Manuals.

[4] Nortek, "Guía rápida - Velocímetro Vectrino Profiler," User Guid., 2013.

[5] Nortek, "Vectrino Profiler Profiling Velocimeter," User Guid., 2012.

[6] D. G. Goring and V. I. Nikora, "Despiking Acoustic Doppler Velocimeter Data," J. Hydraul. Eng., vol. 128, no. 1, pp. 117-126, Jan. 2002, doi: 10.1061/(asce)0733-9429(2002)128:1(117).

[7] B. Khorsandi, L. Mydlarski, and S. Gaskin, "Noise in Turbulence Measurements Using Acoustic Doppler Velocimetry," J. Hydraul. Eng., vol. 138, no. 10, pp. 829-838, Oct. 2012, doi: 10.1061/(asce) hy.1943-7900.0000589.

\section{Autorización y Licencia CC}

Los autores autorizan a APANAC XVIII a publicar el artículo en las actas de la conferencia en Acceso Abierto (Open Access) en diversos formatos digitales (PDF, HTML, EPUB) e integrarlos en diversas plataformas online como repositorios y bases de datos bajo la licencia Attribution-NonCommercialShareAlike 4.0 International (CC BY-NC-SA 4.0) https://creativecommons.org/licenses/by-nc-sa/4.0/.

Ni APANAC XVIII ni los editores son responsables ni del contenido ni de las implicaciones de lo expresado en el artículo. 